\title{
Desenvolvimento e avaliação sensorial de massa de pizza sem glúten, fonte de fibras e adicionada de psyllium
}

\author{
Natália Aparecida Barbosa da Silva ${ }^{1}$, Jucimara Costa Silva ${ }^{2}$, Sabrina Luiza dos Reis Silva ${ }^{3}$, Aline Cristina \\ Arruda Gonçalves ${ }^{3}$, Washington Azevêdo da Silva ${ }^{4}$, Christiano Vieira Pires ${ }^{3}$, Felipe Machado Trombete ${ }^{5^{*}}$
}

\begin{abstract}
Resumo
A demanda dos consumidores brasileiros por produtos de panificação mais saudáveis, que apresentem no rótulo alegações nutricionais, tais como fonte de fibras, tem aumentando nos últimos anos, promovendo também uma maior agregação de valor a tais produtos. Os alimentos sem glúten surgem para atender a população portadora da doença celíaca e também pessoas que não possuem restrição alimentar, mas que optam por consumir esses alimentos por associarem os mesmos com uma alimentação mais saudável. Nessa pesquisa foi avaliada a viabilidade da produção de uma massa de pizza fonte de fibras e sem glúten, adicionada de psyllium, utilizando como base amilácea a farinha de arroz e pasta de inhame. As massas adicionadas de fibras foram comparadas com o controle e avaliadas quanto a aceitação sensorial por escala hedônica e intenção de compra. Para a caracterização dos produtos desenvolvidos também foram realizadas análises físico-químicas, cálculo da composição nutricional e textura instrumental. Verificou-se que, a adição de fibras de psyllium aumentou a extensibilidade da massa crua $(\mathrm{p}<0,05)$ e a firmeza dos discos pré-assados $(\mathrm{p}<0,05)$. A massa com maior concentração de psyllium apresentou os requisitos para ser comercializada no Brasil com a alegação "fonte de fibras", sendo viável a sua produção em maior escala, tanto para públicos celíacos quanto para consumidores que optam por dietas isentas de glúten.
\end{abstract}

Palavras-chave: Panificação. Fibra alimentar. Inhame. Colocasia esculenta. Gluten free.

\section{Development and sensory evaluation of gluten-free pizza dough, source of fiber and added with psyllium}

\begin{abstract}
The demand of Brazilian consumers for healthier bakery products with nutritional claims, such as source of fiber, has increased in recent years, also allowing to add greater commercial value to such products. Gluten-free products are developed to serve the celiac disease population as well as consumers who do not have food restrictions but choose to consume gluten-free products by associating them with healthier food. This work aimed to study the viability of the production of gluten-free and source of fiber pizza dough, added with psyllium, developed with rice flour and yam paste. The dough added of psyllium fibers were compared to the control sample and evaluated for sensory acceptance by the hedonic scale and purchase intention. To characterize the products developed, physicochemical analyzes, nutritional composition and instrumental texture were evaluated. It was found that the addition of psyllium fibers increased the extensibility of the dough $(\mathrm{p}<0,05)$ and also increased the firmness of the pre-baked disks with higher psyllium content $(\mathrm{p}<0,05)$. The formulation with the higher concentration of psyllium presented the requirements to be marketed in Brazil with the claim "source of fibers", being viable its production on a larger scale, both for public celiac and consumers who prefer gluten-free diets.
\end{abstract}

\footnotetext{
${ }^{1}$ Universidade Federal de São João del-Rei. Sete Lagoas, MG. Brasil. https://orcid.org/0000-0002-2836-6069

${ }^{2}$ Universidade Federal de São João del-Rei. Sete Lagoas, MG. Brasil. https://orcid.org/0000-0002-5066-0766

${ }^{3}$ Universidade Federal de São João del-Rei. Sete Lagoas, MG. Brasil. https://orcid.org/0000-0003-4199-5780

${ }^{4}$ Universidade Federal de São João del-Rei. Sete Lagoas, MG. Brasil.

https://orcid.org/0000-0003-1604-7420

${ }^{5}$ Universidade Federal de São João del-Rei. Sete Lagoas, MG. Brasil.

https://orcid.org/0000-0002-8590-4142

*Autor para correspondência: trombete@ufsj.edu.br
}

Recebido para publicação em 01de novembro de 2019. Aceito para publicação em 15 de dezembro de 2019.

e-ISSN: 2447-6218 / ISSN: 2447-6218 / (C) 2009, Universidade Federal de Minas Gerais, Todos os direitos reservados. 
da Silva, N. A. B. et al.

Keywords: Bakery. Dietary fiber. Colocasia esculenta. Gluten-free.

\section{Introdução}

A pizza é um alimento com consumo bastante difundido por todo o mundo e, no Brasil, existe uma demanda de mercado crescente pelas massas prontas para consumo, comercializadas pré-assadas e refrigeradas, principalmente devido a facilidade de preparo e ao baixo custo (Abimapi, 2017). Nos últimos anos, devido a tendência dos consumidores pelo consumo de alimentos mais saudáveis, percebe-se uma diversificação nos tipos de massas de pizzas disponíveis no mercado consumidor, bem como, a realização de pesquisas focadas no desenvolvimento de massas com alegações nutricionais, tais como fonte de fibras, adicionadas de grãos integrais, com adição de leguminosas, prébióticos, isentas de glúten, dentre outros (Glicerina et al., 2017; Han e Koh, 2010 Moore et al., 2009).

A farinha proveniente do trigo é a mais utilizada nos processos de fabricação da massa de pizza, conferindo adequada tenacidade e extensibilidade à massa, no entanto, opções de pizza sem glúten, tais como as formuladas com fécula de mandioca, destinadas ao público celíaco e para consumidores que optam por tais produtos também tem sido pesquisada e já são existentes no mercado (Mazzeo et al., 2015; Gallagher, 2008).

A doença celíaca é uma doença autoimune que atinge indivíduos geneticamente susceptíveis, tendo como principal sinal a alteração das vilosidades da mucosa jejunal. Seu tratamento é basicamente dietético, onde a exclusão total do glúten reflete no desaparecimento dos sintomas manifestados (Jnawali et al., 2016; Farias, 2010). Além do público celíaco, houve um crescimento no consumo de produtos gluten free no Brasil pelo público geral por serem reconhecidos pelos consumidores como mais saudáveis (Ventura et al., 2010).

Durante o desenvolvimento de alimentos isentos de glúten, se faz necessária a substituição do trigo por outros ingredientes. Dentre as alternativas disponíveis, destaca-se o uso de hidrocolóides como a goma xantana, um polissacarídeo produzido por bactérias do gênero Xanthomonas que atua aumentando a retenção de umidade na massa (Nery et al., 2008; Munhoz, 2004). O efeito da adição de fibras de psyllium também tem sido estudado com a mesma finalidade em produtos da panificação, tais como bolos e massas. O psyllium (Plantago ovata) é uma planta arbustiva muito cultivada na Índia, Irã e Paquistão, que cresce geralmente em solo arenoso e salino (Correa et al., 2014) e, suas sementes possuem cerca de 20 a $30 \%$ de mucilagem, sendo muito utilizada pela indústria farmacêutica devido ao elevado teor de fibra solúvel e aos efeitos reconhecidos na redução da absorção de gordura, diminuição dos níveis de colesterol LDL, prevenção da ocorrência de doenças cardiovasculares e constipação (Jovanovski et al., 2018; Dakhara et al., 2012).
O uso do psyllium na panificação sem glúten é uma alternativa para incorporação de fibra alimentar no desenvolvimento desses alimentos, já que, são desenvolvidos geralmente com fécula de mandioca, farinha de arroz, dentre outros produtos amiláceos pobres ou isentos de fibra. A ingestão de alimentos com altos teores de fibras está associada a prevenção de doenças como o câncer, doenças coronarianas, diabetes e redução de colesterol LDL (Veronese et al., 2018). No Brasil, o Ministério da Saúde permite a denominação "fonte de fibras" para os produtos industrializados que possuem no mínimo, 2,5 $\mathrm{g}$ de fibras por porção (Brasil, 2012), devendo conter os dizeres obrigatórios no rótulo: "As fibras alimentares auxiliam o funcionamento do intestino. Seu consumo deve estar associado a uma alimentação equilibrada e hábitos de vida saudáveis" (Brasil, 2018).

Outra alternativa no preparo de alimentos sem glúten é adição de inhame (Colocasia esculenta), também conhecido como taro, uma hortaliça tropical amilácea da família das Araceae, constituída por cerca de 70-80\% de amido (b.s) e muito apreciado pelo sabor suave de seus tubérculos, com elevado valor nutricional, rico em minerais, principalmente potássio e magnésio, vitaminas do complexo B e baixo teor de lipídios (menor que 1\%) (Kaushal et al., 2012, Lewu et al., 2010).

Dessa forma, o presente trabalho teve como objetivo desenvolver uma massa de pizza fonte de fibras e sem glúten, adicionada de fibras de psyllium, utilizando como base amilácea a farinha de arroz e inhame, bem como, avaliar sua aceitabilidade sensorial, características físico-químicas e de textura.

\section{Material e métodos}

\section{Matéria-prima e local das análises}

Os experimentos foram realizados na Universidade Federal de São João del-Rei Campus Sete Lagoas-MG, Departamento de Engenharia de Alimentos. As matérias-primas foram adquiridas no comercio local de Sete Lagoas-MG, sendo apresentadas na Tabela 1. Os inhames foram descascados, lavados, sanificados, cortados e cozidos em água mineral, sendo então produzida a pasta de inhame por esmagamento. Todos os demais ingredientes utilizados foram de uso alimentício, dentro do prazo de validade e, todas as produções foram realizadas com procedimentos de boas práticas de fabricação. 
Desenvolvimento e avaliação sensorial de massa de pizza sem glúten, fonte de fibras e adicionada de psyllium

Tabela 1 - Ingredientes (\%) das formulações de massas de pizza sem glúten (PC) e adicionadas de fibras de psyllium (PAF e PFF)

\begin{tabular}{lccc}
\hline Ingredientes & PC & PAF & PFF \\
\hline Farinha de arroz & $40,1 \%$ & $37,7 \%$ & $35,3 \%$ \\
Inhame & $17,2 \%$ & $16,1 \%$ & $15,1 \%$ \\
Açúcar & $1,8 \%$ & $1,7 \%$ & $1,5 \%$ \\
Água mineral & $14,3 \%$ & $16,1 \%$ & $17,7 \%$ \\
Sal & $1,1 \%$ & $1,0 \%$ & $1,0 \%$ \\
Leite integral & $14,8 \%$ & $14,0 \%$ & $13,0 \%$ \\
Óleo de soja & $2,0 \%$ & $1,9 \%$ & $1,8 \%$ \\
Fermento químico & $2,3 \%$ & $2,1 \%$ & $2,0 \%$ \\
Queijo muçarela & $2,9 \%$ & $2,7 \%$ & $2,5 \%$ \\
Ovo & $2,9 \%$ & $2,7 \%$ & $2,5 \%$ \\
Goma xantana & $0,6 \%$ & $0,00 \%$ & $0,00 \%$ \\
Fibras de psyllium & $0,0 \%$ & $4,0 \%$ & $7,6 \%$ \\
Total & $100 \%$ & $100 \%$ & $100 \%$ \\
\hline
\end{tabular}

Formulações: PC = Pizza controle; PAF = Pizza adicionada de fibras de psyllium; PFF = Pizza fonte de fibras de psyllium.

\section{Desenvolvimento das formulações}

Inicialmente, foram avaliadas diferentes proporções de farinha de arroz, fécula de mandioca, fécula de batata e pasta inhame, a fim de obter um produto com as características adequadas para uma massa de pizza. A formulação definida como ideal teve como base amilácea a farinha de arroz e pasta de inhame. Tal formulação foi considerada como controle (PC), a partir da qual foi elaborada a formulação de pizza adicionada de fibra (PAF), bem como a formulação fonte de fibras (PFF), essa última contendo quantidades de fibras suficiente para ser considerada "Fonte de Fibras" segundo a Resolução RDC no 54, de 12/11/2012 (Brasil, 2012). Na Tabela 1 estão apresentadas as composições das formulações testadas.

O processo de produção das massas pré-assadas ocorreu pela mistura dos ingredientes secos (farinha de arroz, açúcar e sal), seguido da adição da pasta de inhame e do queijo. A essa mistura foram adicionados os ingredientes líquidos (água, leite, óleo e ovo) e, por último, foram incorporados à massa o fermento químico, a goma xantana e as fibras de psyllium de acordo com cada formulação. A massa então foi homogeneizada, dividida e modelada em porções de $80 \mathrm{~g}$, as quais foram assadas em forno a gás por 10 minutos a $180^{\circ} \mathrm{c}$. Os discos pré-assados foram então embalados em sacos de polietileno e armazenados sob refrigeração a $5^{\circ} \mathrm{C}$ até o momento das análises.

\section{Análises físico-químicas}

As massas pré-assadas foram analisadas, em triplicatas, quanto aos seguintes parâmetros físico-químicos: teor de umidade, cinzas, $\mathrm{pH}$, acidez total titulável e cor, utilizando metodologias propostas pelo Instituto Adolfo Lutz (IAL, 2008). A análise de umidade foi realizada em determinador de umidade infravermelho automatizado (OHAUS MB35), a temperatura de $130^{\circ} \mathrm{C}$ até obtenção de peso constante. $\mathrm{O}$ teor de cinzas foi determinado pelo método gravimétrico $(018 / \mathrm{IV})$ em mufla a $550^{\circ} \mathrm{C}$. $\mathrm{O} \mathrm{pH}$ foi determinado por potenciometria, com o auxílio de um pHmetro de bancada (mPA210 MS Tecnopon), conforme método 417/IV. Na análise de acidez total titulável (415/ IV) foram pesados $5 \mathrm{~g}$ de amostra em um Erlenmeyer e então adicionou-se $100 \mathrm{~mL}$ de álcool etílico 95\%, previamente neutralizado e, esta solução foi deixada em repouso por 24 horas, sendo então realizada a titulação com $\mathrm{NaOH}$ $(0,1 \mathrm{M})$ utilizando $20 \mathrm{~mL}$ do sobrenadante, utilizando fenolftaleína como indicador. A cor foi determinada em 10 replicatas por colorimetria digital (CR 400, Konica Minolta, Japão) no sistema CIELAB com escala L*a*b*.

\section{Cálculo da composição nutricional}

A composição nutricional das massas foi calculada por balanço de massa, utilizando o software Dietwin ${ }^{\circledR}$. O software foi alimentado com dados de composição centesimal de cada matéria-prima utilizada, bem como, as quantidades e os rendimentos do processo, gerando então todos os valores obrigatórios na rotulagem nutricional. 
da Silva, N. A. B. et al.

\section{Análise de textura}

Para avaliar as características de textura das massas foram realizadas análises de extensibilidade da massa crua e firmeza da massa assada, ambas em Texturômetro TA.XT ${ }^{\text {Plus }}$ (Stable Micro Systems) (Figura 1), com o auxílio do software Exponent Lite versão 5.1.1.0 licenciada para a UFSJ (2010). Na análise de firmeza foi avaliado o trabalho $(\mathrm{g} / \mathrm{cm})$ necessário para o cisalhamento de um pedaço de massa com $4 \mathrm{~cm}$ de largura e $6 \mathrm{~mm}$ de espessura, utilizando probe de corte $\mathrm{n} \times \mathbf{0} 7$, distância percorrida de $25 \mathrm{~mm}$ e velocidade de $2 \mathrm{~mm} . \mathrm{s}^{-1}$. O teste foi realizado em 9 replicatas. Também foi verificado se a adição de psyllium promoveu aumento da extensibilidade na massa crua, utilizando discos de aproximadamente $12,5 \mathrm{~cm}$ de diâmetro e 5,8 mm de 7 espessura. Os discos com a massa crua foram fixados em plataforma e perfurados com probe esférica SMS P/5S, em movimento perpendicular à massa, na velocidade de $2 \mathrm{~mm} / \mathrm{s}$ até a sua completa ruptura. Nesse teste, quanto mais extensível for a massa maior será a distância percorrida pelo probe, até que seja atingido o limite elástico da massa, causando o rompimento da mesma.

Figura 1 - Fotografia da análise de Extensibilidade da massa de pizza crua (esquerda) e análise de Firmeza na massa de pizza assada (direita) em texturômetro instrumental.

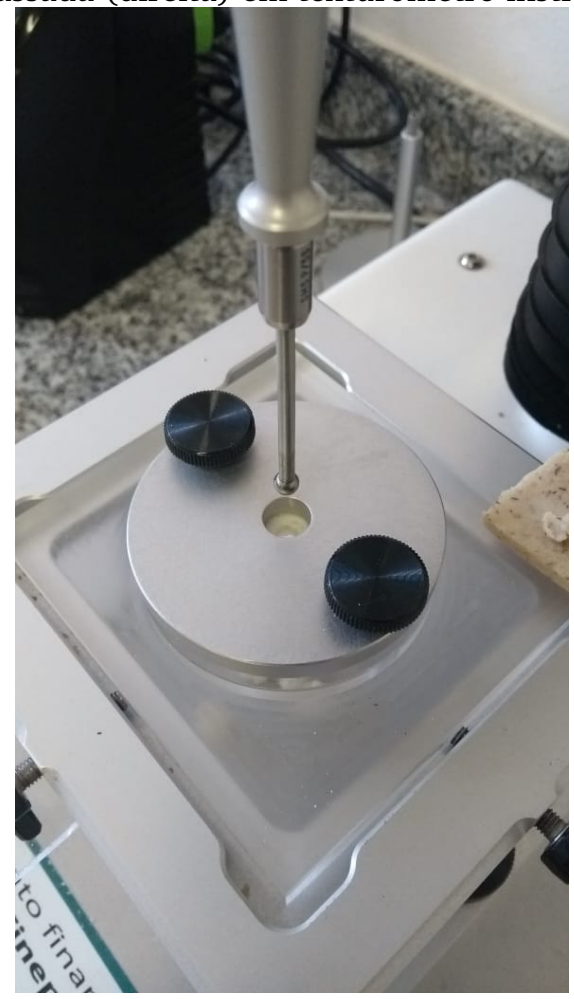

Teste de aceitação e intenção de compra

A análise sensorial foi realizada com 73 avaliadores não treinados, maiores de 18 anos, de ambos os sexos, incluindo professores, alunos e técnicos da UFSJ/ CSL. As massas de pizza (PC, PAF e PFF) foram avaliadas quanto aos atributos cor, aroma, sabor, textura e impressão global, por meio de uma escala hedônica de 9 pontos, com termos variando de "gostei extremamente", nota 9, até "desgostei extremamente", nota 1 (Reis e Minim, 2017). Para tal, os discos das massas pré-assadas foram adicionados de molho de tomate (30 g), queijo muçarela (20 g) e orégano ( $3 \mathrm{~g}$ ) e então submetidas ao forneamento por $180^{\circ} \mathrm{C}$ durante $10 \mathrm{~min}$. Após, foram retiradas do forno e mantidas a $60^{\circ} \mathrm{C}$ até o momento da degustação. As amostras foram servidas em pratos descartáveis codificados com três dígitos, em cabines individuais e sob luz branca (Figura 2). Também foi realizada uma pesquisa de intenção de compra com escala de 5 pontos, variando de

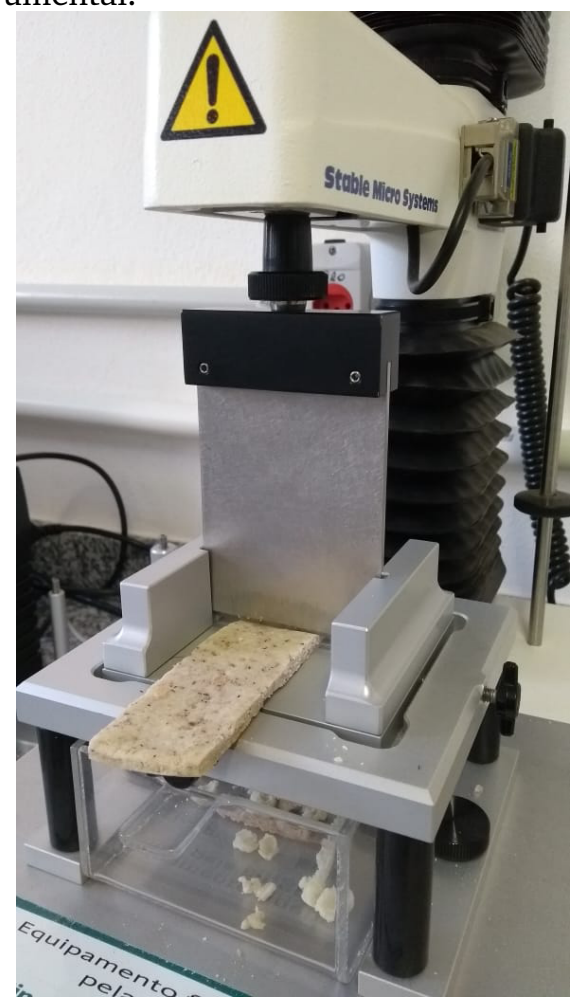

"certamente não compraria este produto" a "certamente compraria este produto".

A realização da análise sensorial teve aprovação do Comitê de Ética da Universidade Federal de São João del-Rei (CAAE 00385318.1.0000.5151).

\section{Análise de dados}

Os dados obtidos foram analisados por estatística descritiva e, para verificar se houve diferença entre os tratamentos estudados, foi realizada Análise de Variância (ANOVA) com comparação das médias pelo Teste de Tukey a nível de 5\% de significância, com o auxílio do software Sisvar 5.6 DEX/UFLA. 
Desenvolvimento e avaliação sensorial de massa de pizza sem glúten, fonte de fibras e adicionada de psyllium

Figura 2 - Fotografias das amostras de pizza sem glúten adicionadas de fibras de psyllium armazenadas a $60^{\circ} \mathrm{C}$ para a análise sensorial (esquerda) e apresentação ao consumidor (direita)

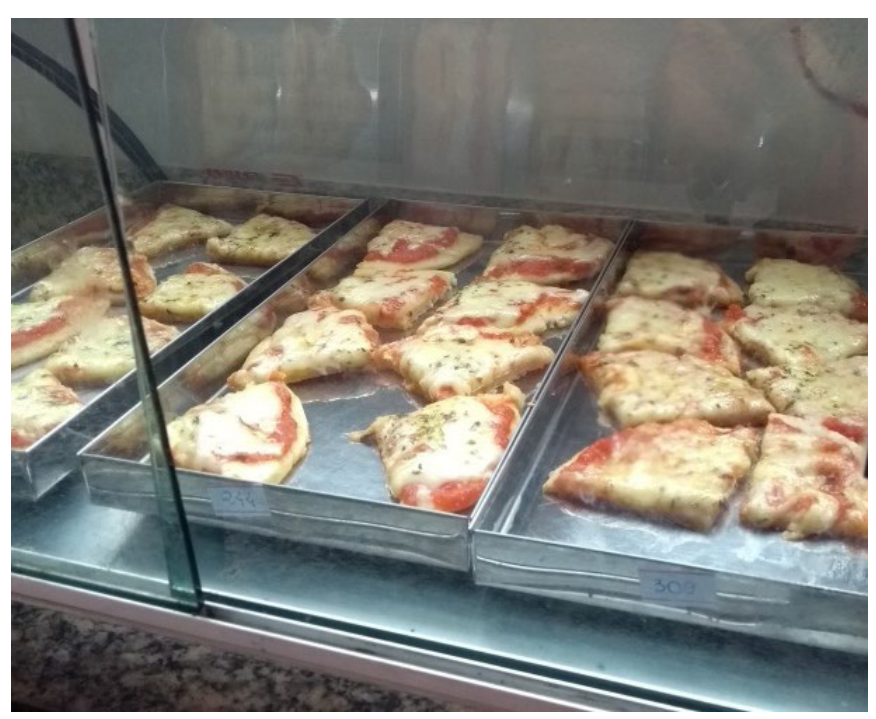

\section{Resultados e discussão}

Os resultados das análises físico-químicas, apresentados na Tabela 2 , demonstraram que não houve diferença significativa $(\mathrm{p}>0,05)$ para os valores de $\mathrm{pH}$, com média geral de $8,33 \pm 0,15 \%$. Na análise de umidade, as amostras PC e PFF diferiram entre si estatisticamente $(\mathrm{p}<0,05)$, sendo a amostra com mais psyllium

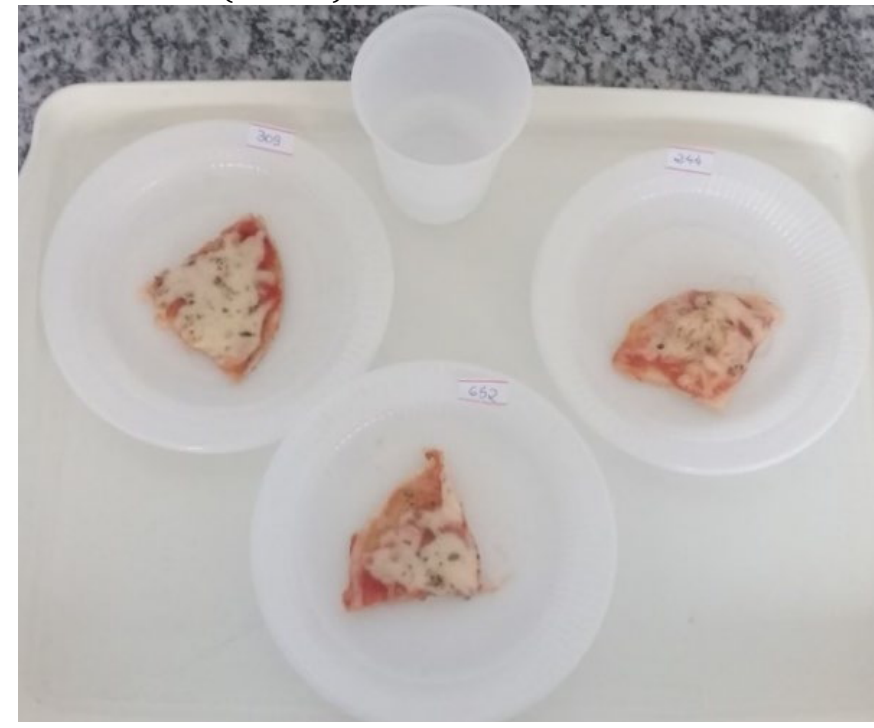

a de maior teor de umidade. Já o teor de cinzas não diferenciou entre as amostras. Fradinho et al. (2015) e Qaisrani et al. (2014) verificaram que quanto maior a adição de fibras de psyllium maior é o teor de minerais nos produtos (em b.s.), podendo ser evidenciado pelo aumento proporcional no teor de cinzas totais, fato que não foi verificado no presente estudo, onde foi utilizado um psyllium com $2,55 \pm 0,04 \%$.

Tabela 2 - Médias \pm DP obtidas das análises físico-químicas de massas de pizzas pré-assadas controle (PC) e com fibras de psyllium (PAF e PFF)

\begin{tabular}{cccc}
\hline Parâmetros & PC & PAF & PFF \\
\hline Umidade (\%) & $33,83^{\mathrm{b}} \pm 0,37$ & $33,60^{\mathrm{b}} \pm 0,30$ & $39,43^{\mathrm{a}} \pm 0,49$ \\
Valor de pH & $8,28^{\mathrm{a}} \pm 0,04$ & $8,50^{\mathrm{a}} \pm 0,21$ & $8,21^{\mathrm{a}} \pm 0,09$ \\
Cinzas (\%) & $3,26^{\mathrm{a}} \pm 0,01$ & $3,14^{\mathrm{b}} \pm 0,00$ & $2,84^{\mathrm{c}} \pm 0,01$ \\
Cor L $^{*}$ & $81,25^{\mathrm{a}} \pm 1,95$ & $76,47^{\mathrm{b}} \pm 0,12$ & $67,86^{\mathrm{c}} \pm 0,40$ \\
Cor a* $^{*}$ & $-0,33^{\mathrm{c}} \pm 0,86$ & $1,85^{\mathrm{b}} \pm 0,36$ & $3,49^{\mathrm{a}} \pm 0,57$ \\
Cor b $^{*}$ & $18,23^{\mathrm{a}} \pm 1,15$ & $19,02^{\mathrm{a}} \pm 0,10$ & $17,81^{\mathrm{a}} \pm 0,47$ \\
\hline
\end{tabular}

Médias seguidas de letras diferentes em cada linha demonstram haver diferença significativa $(\mathrm{p}<0,05)$ entre as amostras. $\mathrm{PC}=\mathrm{Pizza}$ controle $; \mathrm{PAF}$ $=$ Pizza adicionada de fibras; PFF $=$ Pizza fonte de fibras.

Quanto a análise de cor, os parâmetros L* e a* diferiram $(\mathrm{p}<0,05)$ para as três formulações, enquanto o parâmetro b* teve maior valor para a amostra PAF, diferindo das demais. A amostra PFF teve um menor valor de luminosidade, indicando que a amostra é mais escura, fato que não interferiu na aceitação sensorial da mesma quando avaliado pelos provadores na análise sensorial. Quanto ao eixo a* os resultados demonstraram que a amostra PC apresentou um menor valor, indicando leve tendência a cor verde da mesma, fato que não possui relevância prática. Em relação ao eixo b* não houve diferença entre as amostras. Na Figura 3 estão apresentadas as formulações pré-assadas.
Na análise de acidez total titulável, não foi verificado acidez no produto, conforme também verificado pelo $\mathrm{pH}$, que variou de 8,21 a 8,50 . Tais resultados devem-se a utilização do bicarbonato de sódio nas massas, tornando o meio alcalino. A avaliação da extensibilidade das massas demonstrou que a adição de fibras de psyllium tornou a massa mais extensível $(\mathrm{p}<0,05)$, com médias (em força, $\mathrm{g}$ ) de $52,7 \pm 4,7 ; 245,8 \pm 20,8$ e $290,5 \pm 22,9$ para PC, PAF e PFF, respectivamente. Tal fato deve-se as propriedades do psyllium de absorver a água presente na formulação, formando um gel viscoso que altera o comportamento reológico das massas. Quanto a análise de firmeza, todas as amostras também diferiram entre si $(p<0,05)$ sendo a massa pré-assada PFF a que apresentou maior valor. 
da Silva, N. A. B. et al.

As médias obtidas (expresso Kg.seg) corresponderam a $2,6 \pm 0,6 ; 8,6 \pm 2,2$ e 14,3 $\pm 3,1$, respectivamente para as formulações PC, PAF e PFF. Como apresentado nos resultados da avaliação sensorial, a massa PFF foi a que teve melhor aceitação quanto a textura, demonstrando dessa forma que os consumidores preferiram a massa com maior firmeza na mordida.

Figura 3 - Discos de massa de pizza assadas. Da esquerda para direita: massa fonte de fibras (PFF), massa adicionada de fibras (PAF) e massa controle (PC)

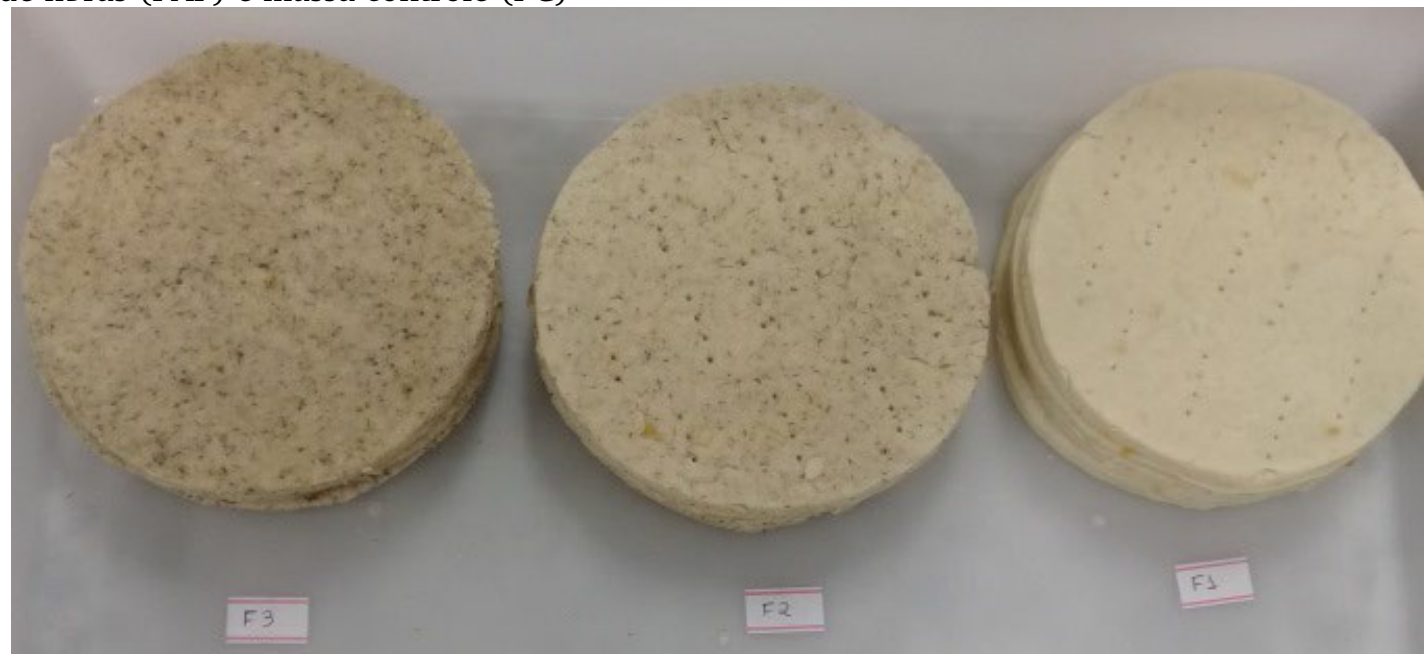

Quanto a composição nutricional das massas (Tabela 3), é possível observar que a formulação PFF possui 3,0 g de fibra alimentar na porção (40 g) e, dessa forma, pode ser considerada uma massa "fonte de fibras" segundo a Resolução RDC no 54, de 12/11/2012 (Brasil, 2012). Como a porção do produto contém cerca de 3,65 $\mathrm{g}$ de psyllium, o produto tem um potencial para receber a alegação de auxiliar na redução da absorção de gordura, devendo conter no rótulo que "seu consumo deve estar associado a uma alimentação equilibrada e hábitos de vida saudáveis" (Brasil, 2018) alcançando os objetivos desse trabalho em desenvolver uma massa de pizza fonte de fibras sem glúten.

Tabela 3 - Resultado da análise de composição nutricional das formulações

\begin{tabular}{|c|c|c|c|c|c|c|}
\hline \multirow[t]{2}{*}{ Parâmetros } & \multicolumn{2}{|c|}{ PC } & \multicolumn{2}{|c|}{ PAF } & \multicolumn{2}{|c|}{ PFF } \\
\hline & $\begin{array}{c}\text { Porção de } 40 \\
g^{*}\end{array}$ & $\% \mathrm{VD}(* *)$ & $\begin{array}{c}\text { Porção de } 40 \\
\mathrm{~g}^{*}\end{array}$ & $\% \mathrm{VD}(* *)$ & $\begin{array}{c}\text { Porção de } 40 \\
g^{*}\end{array}$ & $\begin{array}{c}\% \mathrm{VD} \\
(* *)\end{array}$ \\
\hline Valor Energético & $\begin{array}{c}105,5 \mathrm{Kcal}= \\
440,5 \mathrm{Kj}\end{array}$ & $5,5 \%$ & $\begin{array}{c}86 \mathrm{Kcal}=360 \\
\mathrm{Kj}\end{array}$ & $4 \%$ & $\begin{array}{c}84 \text { Kcal }=349 \\
\mathrm{Kj}\end{array}$ & $4 \%$ \\
\hline Carboidratos & $19,5 \mathrm{~g}$ & $6,5 \%$ & $16 \mathrm{~g}$ & $5 \%$ & $15 \mathrm{~g}$ & $5 \%$ \\
\hline Proteínas & $2,5 \mathrm{~g}$ & $3,5 \%$ & $2,1 \mathrm{~g}$ & $3 \%$ & $2,1 \mathrm{~g}$ & $3 \%$ \\
\hline Gorduras totais & $1,85 \mathrm{~g}$ & $3,5 \%$ & $1,5 \mathrm{~g}$ & $3 \%$ & $1,5 \mathrm{~g}$ & $3 \%$ \\
\hline Gorduras saturadas & $0,5 \mathrm{~g}$ & $2 \%$ & $0,4 \mathrm{~g}$ & $2 \%$ & $0,4 \mathrm{~g}$ & $2 \%$ \\
\hline Gorduras trans & Não contém & $* * *$ & Não contém & $* * *$ & Não contém & $* * *$ \\
\hline $\begin{array}{l}\text { Fibra alimentar } \\
\text { total }\end{array}$ & $0,7 \mathrm{~g}$ & $3 \%$ & $1,7 \mathrm{~g}$ & $7 \%$ & $3,0 \mathrm{~g}$ & $12 \%$ \\
\hline Sódio & $344,5 \mathrm{mg}$ & $14,5 \%$ & $281 \mathrm{mg}$ & $12 \%$ & $269 \mathrm{mg}$ & $11 \%$ \\
\hline
\end{tabular}

*Uma porção equivale a 1/2 unidade. **\% Valores diários com base em uma dieta de 2000 Kcal ou 8400 KJ. Seus valores podem ser maiores ou menores dependendo de suas necessidades energéticas. $\left(^{* *}\right)$ VD não estabelecido. PC $=$ Pizza controle $;$ PAF $=$ Pizza adicionada de fibras; PFF $=$ Pizza fonte de fibras.

Na avaliação sensorial foi verificado que os atributos cor, sabor e aroma não diferiram entre as três formulações analisadas ( $\mathrm{p}>0,05)$, e obtiveram médias gerais equivalentes aos termos "gostei moderadamente" a "gostei muito" (Figura 4). Para o atributo "textura", houve diferença significativa entre as amostras, sendo que, a 
formulação PFF foi a melhor aceita, com nota média de 7,7 ("gostei moderadamente" a "gostei muito") diferindo da formulação controle. Para o atributo "aceitação global", também verificou-se diferença $(\mathrm{p}<0,05)$ referentes à formulação PFF que apresentou médias superiores para todos os atributos citados.

Figura 4 - Médias obtidas na avaliação sensorial, da massa de pizza sem glúten adicionada de fibras de psyllium, utilizando escala hedônica estruturada de 9 pontos para os atributos cor, sabor, aroma, textura e impressão global. PC = Pizza controle; PAF = Pizza adicionada de fibra; PFF = Pizza fonte de fibras. Letras iguais em colunas de um mesmo atributo não diferem entre si $(p>0,05)$ pelo Teste de Tukey

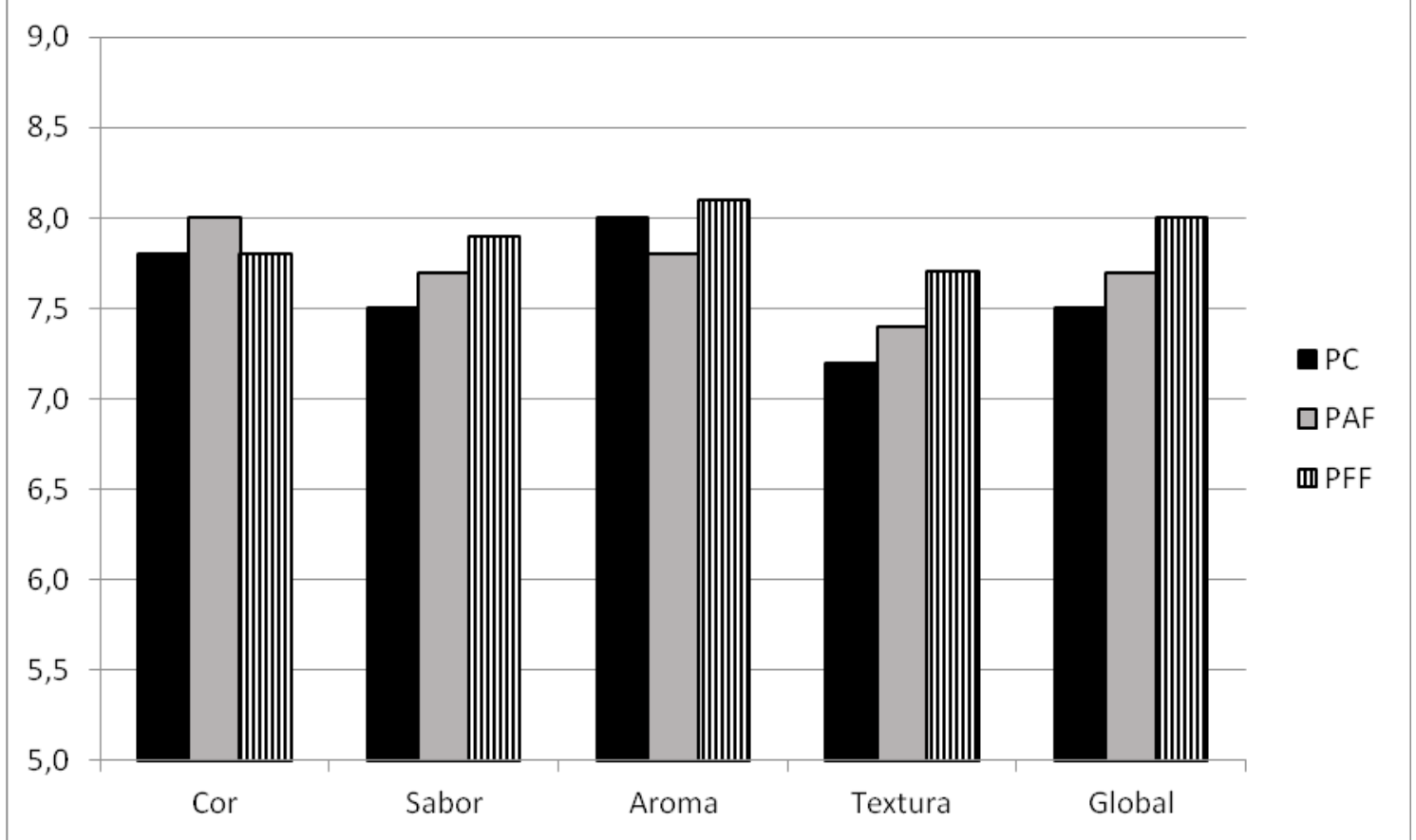

Considerando esses resultados, a adição das fibras de psyllium não influenciou os atributos de cor, aroma e sabor e interferiu positivamente na apreciação da textura e impressão global das massas de pizza, o que demonstra que é possível o desenvolvimento de produtos sem glúten e ao mesmo tempo fonte de fibras, com características sensoriais similares ou até melhores que as dos produtos convencionais. Resultado semelhante foi reportado por Monteiro et al. (2010) que obtiveram médias de aceitação entre 7,0 a 7,3 no atributo "aceitação global" para massas de pizza sem glúten e sem lactose, elaboradas com farinha de arroz, inhame e quinoa.

Quanto a intenção de compra, as formulações PC, PAF e PFF tiveram índices de intenção de compra correspondentes a região positiva na escala utilizada ("provavelmente compraria esse produto" e "certamente compraria o produto"), com escores de 69,8\%, 83,4\% e $87,7 \%$ respectivamente. Portanto, os consumidores expressaram uma confirmação para uma eventual compra de todas as formulações. Isso corrobora mais uma vez para ressaltar o potencial mercadológico dos produtos elaborados, incluindo as adicionadas de fibras.
Destaca-se que o perfil dos avaliadores é de consumidores não celíacos, ou seja, o produto obteve aceitação de um grupo heterogêneo que tem a opção de escolha em consumir ou não um produto gluten free e que possuem paladar acostumados à produtos tradicionais, e mesmo assim apresentou resultados sensoriais satisfatórios.

\section{Conclusão}

Os resultados das análises físico-químicas, de textura e o valor nutricional das massas de pizza pré-assadas, sem glúten, adicionadas de fibra ou fonte de fibras, desenvolvidas nessa pesquisa, demonstram que os produtos têm um potencial tecnológico para ser explorado industrialmente como produtos de melhor valor nutricional e de características diferenciadas. Todas as formulações obtiveram boa aceitação sensorial e ótimo índice de intenção de compra, permitindo concluir que é viável a utilização da farinha de arroz, pasta de inhame e fibras de psyllium na elaboração do produto em conformidade com a legislação para ser comercializado tanto como adicionado de fibra ou como "fonte de fibra". O produto pode ser consumido por portadores de doença celíaca, desde que se evite a contaminação cruzada com outras preparações que possuam glúten em sua composição. 


\section{Referências}

Abimapi - Associação Brasileira das Indústrias de Biscoitos, Massas Alimentícias e Pães \& Bolos Industrializados. Estatísticas da produção nacional. 2017. Disponível em: https://www.abimapi.com.br/.

Brasil. 2018. Ministério da Saúde. Agência Nacional de Vigilância Sanitária. Alegações de propriedade funcional aprovadas. Disponível em http://portal.anvisa.gov.br.

Brasil. 2012. Ministério da Saúde. RDC no 54, de 12 de novembro de 2012. Regulamento Técnico sobre Informação Nutricional Complementar. Diário Oficial da União. Disponível em: http://portal. anvisa.gov.br/documents/\%2033880/2568070/rdc0054_12_11_2012. pdf/c5ac23fd-974e-4f2c-9fbc-48f7e0a31864/.

Correa, A. C. 2014. Perfil sensorial e direcionadores de preferência em bebida de caju (Anacardium ocidentale L.) com finalidade dietética, adicionada de psyllium. Dissertação (Mestrado em Alimentos e Nutrição). Universidade Estadual de Campinas. 124 f. Disponível em: http://repositorio.unicamp.br/jspui/handle/REPOSIP/254242.

Dakhara, S. L.; Anajwala, C. C.; Selote, V. S. 2012. Fibrous drugs for curing various common health problems. Pharmacognosy Reviews, 6: 16-21. Doi: https://dx.doi.org/10.4103/0973-7847.95853.

Farias, A. D. S. 2010. Massas para pizza com restrição de glúten. 2009. Monografia (Especialização em Qualidade de Alimentos). Universidade de Brasília. $41 \mathrm{f}$.

Fradinho, P.; Nunes, M. C.; Raymundo, A. 2015. Developing consumer acceptable biscuits enriched with Psyllium fiber. Journal of food science and technology, 52: 4830-4840. Doi: https://dx.doi.org/10.1007/ s13197-014-1549-6.

Gallagher, E. 2008. Formulation and nutritional aspects of gluten-free cereal products and infant foods. In: Gluten-free cereal products and beverages, 321-346. Academic Press.

Glicerina, V.; Balestra, F.; Capozzi, F.; Dalla Rosa, M.; Romani, S. 2018. Influence of the addition of soy product and wheat fiber on rheological, textural, and other quality characteristics of pizza. Journal of Texture Studies, 49: 415-423. Doi: https://dx.doi.org/10.1111/jtxs.12311.

Han, H. M.; Koh, B. K. 2011. Antioxidant activity of hard wheat flour, dough and bread prepared using various processes with the addition of different phenolic acids. Journal of the Science of Food and Agriculture, 91: 604-608. Doi: https://dx.doi.org/10.1002/jsfa.4188.

Instituto Adolfo Lutz. 2008. Métodos físico-químicos para análise de alimentos. Ribeirão Preto: Instituto Adolfo Lutz. São Paulo.

Jnawali, P.; Kumar, V.; Tanwar, B. 2016. Celiac disease: Overview and considerations for development of gluten-free foods. Food Science and Human Wellness, 5: 169-176. Doi: https://doi.org/10.1016/j. fshw.2016.09.003.
Kaushal, P.; Kumar, V.; Sharma, H. K. 2012. Comparative study of physicochemical, functional, antinutritional and pasting properties of taro (Colocasia esculenta), rice (Oryza sativa) flour, pigeonpea (Cajanus cajan) flour and their blends. LWT-Food Science and Technology, 48: 59-68. Doi: https://doi.org/10.1016/j.lwt.2012.02.028.

Lewu, M. N.; Adebola, P. O.; Afolayan, A. J. 2010. Effect of cooking on the mineral contents and anti-nutritional factors in seven accessions of Colocasia esculenta (L.) Schott growing in South Africa. Journal of Food Composition and Analysis, 23: 389-393. Doi: https://doi. org/10.1016/j.jfca.2010.02.006.

Mazzeo, T.; Cauzzi, S.; Brighenti, F.; Pellegrini, N. 2015. The development of a composition database of gluten-free products. Public Health Nnutrition, 18: 1353-1357. Doi: https://dx.doi.org/10.1017/ S1368980014001682.

Monteiro, S. Z. 2013. Utilização de mesclas de farinhas de arroz, Inhame e quinoa na elaboração de disco de pizza pré assado sem glúten e sem lactose. Trabalho de Conclusão de Curso (Engenharia de Alimentos). Universidade Federal do Rio Grande do Sul. 82f. Disponível em: http:// hdl.handle.net/10183/87552.

Moore, J.; Luther, M.; Cheng, Z.; Yu, L. 2009. Effects of baking conditions, dough fermentation, and bran particle size on antioxidant properties of whole-wheat pizza crusts. Journal of Agricultural and Food Chemistry, 57: 832-839. Doi: https://dx.doi.org/10.1021/jf802083x.

Munhoz, M. P.; Weber, F. H.; Chang, Y. K. 2004. Influência de hidrocolóides na textura de gel de amido de milho. Ciência e Tecnologia de Alimentos, 24: 403-406. Doi: http://dx.doi.org/10.1590/S010120612004000300018.

Nery, T. B. R.; Brandão, L. V.; Esperidião, M. C. A.; Druzian, J. I. 2008. Biossíntese de goma xantana a partir da fermentação de soro de leite: rendimento e viscosidade. Química Nova, 31: 1937-1941. Doi: http:// dx.doi.org/10.1590/S0100-40422008000800004.

Qaisrani, T. B.; Butt, M. S., Hussain; S.; Ibrahim, M. 2014. Characterization and utilization of psyllium husk for the preparation of dietetic cookies. International Journal of Modern Agriculture, 3: 81-91.

Ventura, R. 2010. Mudanças no perfil do consumo no Brasil: principais tendências nos próximos 20 anos. Rio de Janeiro: Macroplan.

Veronese, N., Solmi, M., Caruso, M. G., Giannelli, G., Osella, A. R., Evangelou, E.; Tzoulaki, I. 2018. Dietary fiber and health outcomes: an umbrella review of systematic reviews and meta-analyses. The American journal of clinical nutrition, 107(3), 436-444. Doi: https:// dx.doi.org/10.1093/ajcn/nqx082 . 